\title{
System-Level Performance Analysis in 3D Drone Mobile Networks
}

\author{
Jiayi Huang ${ }^{1}$, Arman Shojaeifard ${ }^{2}$, Jie Tang ${ }^{1}$, and Kai-Kit Wong ${ }^{3}$ \\ 1 South China University of Technology, Guangzhou, China \\ 2 BT Labs, Adastral Park, Ipswich IP5 3RE, UK arman. shojaeifard@bt.com \\ 3 University College London, London WC1E 7JE, UK \\ kai-kit.wong@ucl.ac.uk
}

\begin{abstract}
We present a system-level anaysis for drone mobile networks on a finite three-dimensional (3D) space. A performance boundary derived by deterministic random (Brownian) motion model over Nakagami$\mathrm{m}$ fading interfering channels is developed. This method allows us to circumvent the extremely complex reality model and obtain the upper and lower performance bounds of actual drone mobile networks. The validity and advantages of the proposed framework are confirmed via extensive Monte-Carlo(MC) simulations. The results reveal several important trends and design guidelines for the practical deployment of drone mobile networks.
\end{abstract}

Keywords: Drone cellular networks $\cdot$ system-level analysis $\cdot$ stochastic geometry theory performance boundary - Monter-Carlo (MC) simulations.

\section{Introduction}

The exponential growth of wireless data services driven by mobile smart devices (e.g., smartphone, pad) has triggered the investigation of assisted terrestrial 
Jiayi Huang et al.

networks in the era of the Internet of Things (IoT) [1]. However, it's a crucial task under the circumstances of large-gatherings (e.g., sports games, concerts) and natural or man-made disasters (e.g., floods, earthquake).Due to the advantages of Unmanned aerial vehicles (UAVs), UAVs assisted cellular network is considered a prominent solution for enhancing or recovering terrestrial cellular networks, which has attracted great attention in both academia and industry recently.Therefore, the fifth-generation (5G) communication system also considers the application of low-altitude drones in the system [2].

Among current wireless network researches, there are many published works related to the UAVs assisted networks, many works in the literature consider simplified movement models (e.g., fixed height around a circle). However, the reality is more complicated than these models and we can only obtain drone system performance in simple cases using these models. In [3], Poisson cluster process was applied to distribute user and drone hover in a certain height above cluster center in order to compare the performance of millimeter wave (mmWave) and sub-6 GHz. The work in [4] converted the problem of three-dimensional space into two-dimensional plane by distributing drones in a fixed altitude. In [5] the authors concluded that a cellular network with an omnidirectional antenna can support drone base stations downlinks and control channels in a low altitude, but a high altitude is still struggle. The authors in [6] investigated the coverage probability and average achievable rate in the post-disaster area by using two cooperative drones in a fixed height. This paper provides a unifed model for performance analysis of drone mobile networks and obtain the lower and upper bounds of actual drone mobile networks by introduced deterministic motion model and 3D Brownian motion model. We explicitly account for certain constraints, such as small-scale and large-scale fading characteristics depending on line-of-sight (LOS) and non line-of-sight (NLOS) propagation, and the impact 
System-Level Performance Analysis in 3D Drone Mobile Networks

of drone mobility based on 3D deterministic random (Brownian) motion. The analytical formulations are validated via Monter-Carlo (MC) simulations.

\section{Notation}

$\boldsymbol{X}$ is a matrix; $\boldsymbol{x}$ is a vector; $T, \dagger$, and + are the transpose, Hermitian, and pseudo-inverse operations; $\mathbb{E}_{x}[$.$] is the expectation; \operatorname{Pr}[$.$] is the probability; \mathcal{F}_{x}[$. is the cumulative distribution function $(\mathrm{CDF}) ; \mathcal{P}_{x}[$.$] is the probability density$ function $(\mathrm{PDF}) ; \mathcal{L}_{x}[$.$] is the Laplace transform (LT) function; |x|$ is the modulus; $\|\boldsymbol{x}\|$ is the Euclidean norm; $\mathbf{I}_{(.)}$is the identity matrix; $\mathcal{H}($.$) is the Heaviside$ step function; $\delta($.$) is the Delta function; \mathcal{C N}\left(\mu, \nu^{2}\right)$ is the circularly-symmetric complex Gaussian distribution with mean $\mu$ and variance $\nu^{2} ; \Gamma($.$) and \Gamma(.,$.$) are$ the Gamma and incomplete (upper) Gamma functions; $\mathcal{G}(\kappa, \theta)$ is the Gamma distribution with shape parameter $\kappa$ and scale parameter $\theta$, respectively.

\section{Preliminaries}

In this word, we consider a large-scale UAV network in which K BSs (i.e., drones) are deployed on the finite $3 \mathrm{D}$ ball of radius $\mathrm{R}$ according to a homogeneous PPP $\Phi$ with spatial density $\lambda$ at time $t=0$. Let $\mathrm{K}_{\mathrm{LOS}}$ and $\mathrm{K}_{\mathrm{NLOS}}$ respectively denote the number of drones experiencing LOS and NLOS propagation (i.e., $\mathrm{K}=\mathrm{K}_{\mathrm{LOS}}+$ $\mathrm{K}_{\mathrm{NLOS}}$ ) at time $t=0$. Based on the stationary property of PPP, and Slivnyak's theorem [7], the analysis is carried for a typical user $o$ assumed to be located at the origin.

The drones, equipped with $\mathrm{M}$ transmit antennas, are considered to be serving a user per resource block. Let $\boldsymbol{h}_{b}^{T} \sim \mathcal{C N}\left(\mathbf{0}, \mathbf{I}_{\mathrm{M}}\right)$ denote the small-scale fading channel between the typical user $o$ and its serving drone $b$. Here, we utilize the Nakagami- $m$ distribution, which can capture a wide range of small-scale fading conditions through tuning of the parameter $m$. Considering the drones 
Jiayi Huang et al.

apply conjugate-beamforming (CB), the intended (from drone $b$ ) small-scale fading channel power gains under LOS and NLOS propagation are distributed as $g_{b} \sim \mathcal{G}\left(m \mathrm{M}, \frac{1}{m}\right)$ and $g_{b} \sim \mathcal{G}\left(\mathrm{M}, \frac{1}{m}\right)$, respectively. The interfering (from drone $i$ ) small-scale fading channel power gains under LOS and NLOS propagation are distributed as $g_{i} \sim \mathcal{G}\left(m, \frac{1}{m}\right)$ and $g_{i} \sim \mathcal{G}(1,1)$, respectively [8].

The path-loss function is defined as

$$
L(r)=\max \left(\beta_{0}, \beta_{1} r^{-\alpha}\right)
$$

where $r$ is the distance, $\beta_{0}$ is the minimum coupling loss, $\beta_{1}$ is a constant parameter of the path-loss function, and $\alpha$ is the path-loss exponent. All channels undergo free-space path-loss, i.e., $\alpha=2$. Moreover, $\beta_{1}=\frac{1}{\epsilon}\left(\frac{c}{4 \pi f_{c}}\right)^{2}$, where $c=3 \times 10^{8} \mathrm{~m} / \mathrm{s}$ is the light speed, and $f_{c}$ is the carrier frequency. For LOS $\left(L_{\mathrm{LOS}}(r)\right)$ and $\operatorname{NLOS}\left(L_{\mathrm{NLOS}}(r)\right)$ links, $\epsilon=\epsilon_{\mathrm{LOS}}=1 \mathrm{~dB}$ and $\epsilon=\epsilon_{\mathrm{NLOS}}=20$ $\mathrm{dB}$, respectively [9].

We utilize the following function for the probability of LOS propagation

$$
\operatorname{Pr}\left[\mathrm{LOS}, r_{(.)}=r\right]= \begin{cases}1 & \text { if } r \in[0, \mathrm{D}) \\ 0 & \text { if } r \in[\mathrm{D}, \mathrm{R}]\end{cases}
$$

where $\mathrm{D}$ denotes the critical distance. Under this model, given the drones follow from a homogeneous PPP $\Phi$, the average number of LOS and NLOS drones in a $3 \mathrm{D}$ ball of radius $\mathrm{R}$ are respectively $\overline{\mathrm{K}}_{\mathrm{LOS}}=\frac{4}{3} \pi \mathrm{D}^{3} \lambda$ and $\overline{\mathrm{K}}_{\mathrm{NLOS}}=\frac{4}{3} \pi\left(\mathrm{R}^{3}-\mathrm{D}^{3}\right) \lambda$.

We consider the cellular association strategy in which the typical user $o$ connects to the drone $b$ which provides the greatest received SINR.

Lemma 1 Considering there are $\mathrm{K}_{\mathrm{LOS}}$ LOS drones (i.e., $\mathrm{K}_{\mathrm{LOS}}>0$ ) uniformlydeployed on the finite $3 \mathrm{D}$ ball of radius $\mathrm{D}$, the $\mathrm{CDF}$ and PDF of the distance between the typical user $o$ and its serving LOS drone, $r_{b, \mathrm{LOS}}$, are respectively 
System-Level Performance Analysis in 3D Drone Mobile Networks

given by

$$
\begin{gathered}
\mathcal{F}_{r_{b, \mathrm{LOS}}}(r)=1-\left(1-\frac{r^{3}}{\mathrm{D}^{3}}\right)^{\mathrm{K}_{\mathrm{LOS}}}, \quad 0 \leq r \leq \mathrm{D} \\
\mathcal{P}_{r_{b, \mathrm{LOS}}}(r)=\frac{3 r^{2} \mathrm{~K}_{\mathrm{LOS}}}{\mathrm{D}^{3}}\left(1-\frac{r^{3}}{\mathrm{D}^{3}}\right)^{\mathrm{K}_{\mathrm{LOS}}-1}, \quad 0 \leq r \leq \mathrm{D} .
\end{gathered}
$$

Proof: The result follows from [10, Theorem 2.1] with $n=1, d=3$.

Lemma 2 Considering there are $\mathrm{K}_{\mathrm{NLOS}}$ NLOS drones (i.e., $\mathrm{K}_{\mathrm{LOS}}=0$ ) uniformlydeployed on the finite $3 \mathrm{D}$ ball double-bounded by radii $\mathrm{D}(<\mathrm{R})$ and $\mathrm{R}$, the $\mathrm{CDF}$ and PDF of the distance between the typical user $o$ and its serving NLOS drone, $r_{b, \mathrm{NLOS}}$, are respectively given by

$$
\begin{gathered}
\mathcal{F}_{r_{b, \mathrm{NLOS}}}(r)=1-\left(1-\frac{r^{3}-\mathrm{D}^{3}}{\mathrm{R}^{3}-\mathrm{D}^{3}}\right)^{\mathrm{K}_{\mathrm{NLOS}}}, \quad \mathrm{D} \leq r \leq \mathrm{R} \\
\mathcal{P}_{r_{b, \mathrm{NLOS}}}(r)=\frac{3 r^{2} \mathrm{~K}_{\mathrm{NLOS}}}{\mathrm{R}^{3}-\mathrm{D}^{3}}\left(1-\frac{r^{3}-\mathrm{D}^{3}}{\mathrm{R}^{3}-\mathrm{D}^{3}}\right)^{\mathrm{K}_{\mathrm{NLOS}}-1}, \quad \mathrm{D} \leq r \leq \mathrm{R} .
\end{gathered}
$$

Proof: The proof, ommitted due to space limitations, follows from the probability distribution of a double-bounded random process given in [11, Eqn. (3)].

\section{Unified Framework}

In this section, we present a stochastic geometry-based model for performance analysis of drone mobile networks. By introducing Slivnyak's theorem, the analysis is carried for a typical user $o$ assumed to be located at the origin. 
Jiayi Huang et al.

\subsection{D Brownian Motion}

The drones are considered to be mobile according to a 3D Brownian motion $(\mathrm{BM})$. At time $t>0$, the movement of an arbitrary drone can be captured through the following stochastic differential equation (SDE) [12].

$$
\mathrm{d} l(t)=\sigma \mathrm{d} b(t)
$$

where $l(t)=\left\{l_{x}(t), l_{y}(t), l_{z}(t)\right\}$ is a vector for the Cartesian coordinates at time $t, b(t)=\left\{b_{x}(t), b_{y}(t), b_{z}(t)\right\}$ represents the standard BM (i.e., Wiener process) vector at time $t$, and $\sigma$ is a positive constant (e.g., representing average velocity). Here, we consider $b_{x}(t), b_{y}(t), b_{z}(t) \sim \mathcal{N}(0, t)$. The corresponding Euclidean distance with respect to the origin at time $t$ can be formulated as $\hat{r}(t)=\sqrt{l_{x}^{2}(t)+l_{y}^{2}(t)+l_{z}^{2}(t)}$. Here, the mobility model should account for the finite volume of the 3D ball as well as the LOS/NLOS propagation conditions. Hence, we consider the case where (i) the mobile LOS drone cannot be at a distance larger than $\mathrm{D}$ with respect to the origin at any given time, i.e., $r_{\mathrm{LOS}}(t)=\max (0, \min (\mathrm{D}, \hat{r}(t)))$, and (ii) the mobile NLOS drone cannot be at a distance smaller than $D$ and larger than $R$ with respect to the origin at any given time, i.e., $r_{\mathrm{NLOS}}(t)=\max (\mathrm{D}, \min (\mathrm{R}, \hat{r}(t)))$.

Lemma 3 With the 3D BM mobility model under consideration, the CDF and PDF of the mobile LOS drone distance with respect to the origin at time $t$ (i.e., $\left.r_{\mathrm{LOS}}(t)=\max (0, \min (\mathrm{D}, \hat{r}(t)))\right)$ are respectively given by

$$
\mathcal{F}_{r_{\mathrm{LOS}}(t)}(w)=\left(1+\frac{2}{\sqrt{\pi}} \Gamma\left(\frac{3}{2}, \frac{w^{2}}{4 \sigma t}\right)(\mathcal{H}(w-\mathrm{D})-1)\right) \mathcal{H}(w)
$$

and

$$
\mathcal{P}_{r_{\mathrm{LOS}}(t)}(w)=\left(1+\frac{2}{\sqrt{\pi}} \Gamma\left(\frac{3}{2}, \frac{w^{2}}{4 \sigma t}\right)(\mathcal{H}(w-\mathrm{D})-1)\right) \delta(w)+
$$


System-Level Performance Analysis in 3D Drone Mobile Networks

$$
\left(\frac{2}{\sqrt{\pi}} \Gamma\left(\frac{3}{2}, \frac{w^{2}}{4 \sigma t}\right) \delta(w-\mathrm{D})-\frac{w^{2}}{2 \sqrt{\pi}(\sigma t)^{\frac{3}{2}}} \exp \left(-\frac{w^{2}}{4 \sigma t}\right)(\mathcal{H}(w-\mathrm{D})-1)\right) \mathcal{H}(w) .
$$

Lemma 4 With the 3D BM mobility model under consideration, the CDF and PDF of the mobile NLOS drone distance with respect to the origin at time $t$ (i.e., $\left.r_{\mathrm{NLOS}}(t)=\max (\mathrm{D}, \min (\mathrm{R}, \hat{r}(t)))\right)$ are respectively given by

$$
\mathcal{F}_{r_{\mathrm{NLOS}}(t)}(w)=\left(1+\frac{2}{\sqrt{\pi}} \Gamma\left(\frac{3}{2}, \frac{w^{2}}{4 \sigma t}\right)(\mathcal{H}(w-\mathrm{R})-1)\right) \mathcal{H}(w-\mathrm{D})
$$

and

$$
\begin{aligned}
\mathcal{P}_{r_{\mathrm{NLOS}}(t)}(w)= & \left(1+\frac{2}{\sqrt{\pi}} \Gamma\left(\frac{3}{2}, \frac{w^{2}}{4 \sigma t}\right)(\mathcal{H}(w-\mathrm{R})-1)\right) \delta(w-\mathrm{D}) \\
& +\left(\frac{2}{\sqrt{\pi}} \Gamma\left(\frac{3}{2}, \frac{w^{2}}{4 \sigma t}\right) \delta(w-\mathrm{R})\right) \mathcal{H}(w-\mathrm{D}) \\
& -\left(\frac{1}{2 \sqrt{\pi} w}\left(\frac{w^{2}}{\sigma t}\right)^{\frac{3}{2}} \exp \left(-\frac{w^{2}}{4 \sigma t}\right)(\mathcal{H}(w-\mathrm{R})-1)\right) \mathcal{H}(w-\mathrm{D}) .
\end{aligned}
$$

Next, we aim to characterize the distribution of the reference transmitterreceiver distance based on the 3D BM mobility and LOS/NLOS propagation models under consideration.

Corollary 1 The closest transmitter-receiver distance for the serving LOS drone becomes equivalent to the 3D BM mobility model with the following drones spatial density [13]

$$
\lambda=\frac{3 \log \left(1-\frac{\mathcal{H}(w)}{\sqrt{\pi}}\left(2 \mathcal{H}(w-D) \Gamma\left(\frac{3}{2}, \frac{w^{2}}{4 t \sigma}\right)-2 \Gamma\left(\frac{3}{2}, \frac{w^{2}}{4 t \sigma}\right)+\sqrt{\pi}\right)\right)}{4 \pi D^{3} \log \left(1-\frac{w^{3}}{D^{3}}\right)}
$$

Corollary 2 The closest transmitter-receiver distance for the serving NLOS drone becomes equivalent to the 3D BM mobility model with the following drones 
Jiayi Huang et al.

spatial density

$$
\lambda=\frac{3 \log \left(1-\frac{\mathcal{H}(w-\mathrm{D})}{\sqrt{\pi}}\left(2 \mathcal{H}(w-\mathrm{R}) \Gamma\left(\frac{3}{2}, \frac{w^{2}}{4 t \sigma}\right)-2 \Gamma\left(\frac{3}{2}, \frac{w^{2}}{4 \sigma t}\right)+\sqrt{\pi}\right)\right)}{4 \pi\left(\mathrm{R}^{3}-\mathrm{D}^{3}\right) \log \left(1-\frac{w^{3}-\mathrm{D}^{3}}{R^{3}-\mathrm{D}^{3}}\right)} .
$$

\subsection{Deterministic Motion}

Next, we consider the case where the movement of the drones is deterministic such that they move at a constant speed towards a target. At time $t>0$, the corresponding Euclidean distance with respect to the origin is given by

$$
\hat{r}(t)=r_{0}-v t
$$

where $r_{0}$ is the distance at time $t=0$ and $v$ is the constant speed, respectively. Note that a negative value for $v$ indicates movement in the opposite direction and vice versa. Here, we need to account for the finite volume of the $3 \mathrm{D}$ ball as well as the LOS/NLOS propagation conditions. Hence, we consider the case where (i) the mobile LOS drone cannot be at a distance larger than $\mathrm{R}$ with respect to the origin at any given time, i.e., $r_{\mathrm{LOS}}(t)=\max (0, \min (\mathrm{D}, \hat{r}(t)))$, and (ii) the mobile NLOS drone cannot be at a distance smaller than $\mathrm{D}$ and larger than $\mathrm{R}$ with respect to the origin at any given time, i.e., $r_{\mathrm{NLOS}}(t)=\max (\mathrm{D}, \min (\mathrm{R}, \hat{r}(t)))$.

Lemma 5 With the deterministic mobility model under consideration, the CDF and PDF of the mobile LOS drone distance with respect to the origin at time $t$ (i.e., $\left.r_{\mathrm{LOS}}(t)=\max (0, \min (\mathrm{D}, \hat{r}(t)))\right)$ are respectively given by

$$
\mathcal{F}_{r_{\mathrm{LOS}}(t)}(w)=\mathcal{H}(w)\left(1+(\mathcal{H}(w-\mathrm{D})-1)\left(1-\frac{(v t+w)^{3}}{\mathrm{D}^{3}}\right)^{\mathrm{K}_{\mathrm{LOS}}}\right)
$$

and

$\mathcal{P}_{r_{\mathrm{LOS}}(t)}(w)=\delta(w)+(\mathcal{H}(w) \delta(w-\mathrm{D})+(\mathcal{H}(w-\mathrm{D})-1) \delta(w))\left(1-\frac{(v t+w)^{3}}{\mathrm{D}^{3}}\right)^{\mathrm{K}_{\mathrm{LOS}}}$ 
System-Level Performance Analysis in 3D Drone Mobile Networks

$$
-\mathcal{H}(w)(\mathcal{H}(w-\mathrm{D})-1) \frac{3 \mathrm{~K}_{\mathrm{LOS}}(v t+w)^{2}}{\mathrm{D}^{3}}\left(1-\frac{(v t+w)^{3}}{\mathrm{D}^{3}}\right)^{\mathrm{K}_{\mathrm{LOS}}-1} .
$$

Lemma 6 With the deterministic mobility model under consideration, the CDF and PDF of the mobile NLOS drone distance with respect to the origin at time $t$ (i.e., $\left.r_{\mathrm{NLOS}}(t)=\max (\mathrm{D}, \min (\mathrm{R}, \hat{r}(t)))\right)$ are respectively given by

$$
\mathcal{F}_{r_{\mathrm{NLOS}}(t)}(w)=\mathcal{H}(w-\mathrm{D})\left(1+(\mathcal{H}(w-\mathrm{R})-1)\left(\frac{\mathrm{R}^{3}-(v t+w)^{3}}{\mathrm{R}^{3}-\mathrm{D}^{3}}\right)^{\mathrm{K}_{\mathrm{NLOS}}}\right)
$$

and

$$
\begin{aligned}
\mathcal{P}_{r_{\mathrm{NLOS}}(t)}(w)= & \delta(w-\mathrm{D})\left(1+(\mathcal{H}(w-\mathrm{R})-1)\left(\frac{\mathrm{R}^{3}-(v t+w)^{3}}{\mathrm{R}^{3}-\mathrm{D}^{3}}\right)^{\mathrm{K}_{\mathrm{NLOS}}}\right) \\
& +\mathcal{H}(w-\mathrm{D})\left(\delta(w-\mathrm{D})\left(\frac{\mathrm{R}^{3}-(v t+w)^{3}}{\mathrm{R}^{3}-(\mathcal{H}(w-\mathrm{R})-1) \mathrm{D}^{3}}\right)^{\mathrm{K}_{\mathrm{NLOS}}}\right) \\
& -\mathcal{H}(w-\mathrm{D})\left(\frac{3 \mathrm{~K}_{\mathrm{NLOS}}(v t+w)^{2}}{\mathrm{R}^{3}-\mathrm{D}^{3}}\left(\frac{\mathrm{R}^{3}-(t v+w)^{3}}{\mathrm{R}^{3}-\mathrm{D}^{3}}\right)^{\mathrm{K}_{\mathrm{NLOS}}-1}\right)
\end{aligned}
$$

\subsection{SINR Formulation}

The received SINR at the reference user $o$ is given by

$$
\mathrm{SINR}=\frac{X}{I+\sigma^{2}}
$$

where

$$
X=p g_{b} L\left(r_{b}\right)
$$


Jiayi Huang et al.

and

$$
I=\sum_{i \in \Phi \backslash\{b\}} p g_{i} L\left(r_{i}\right)
$$

with $p$ and $\sigma^{2}$ respectively used to denote the transmit power and noise variance.

\subsection{SE Formulation}

Theorem 1. The average rate (in nat $/ \mathrm{s} / \mathrm{Hz}$ ) of the typical user is given by

$$
\mathbb{E}[\log (1+\operatorname{SINR})]=\int_{0}^{+\infty} \int_{0}^{+\infty} \frac{1-\mathcal{F}_{\text {SINR } \mid r_{b}=r}[\gamma]}{1+\gamma} \mathrm{d} \gamma \mathcal{P}_{r_{b}}(r) \mathrm{d} r
$$

where $\mathcal{F}_{\mathrm{SINR} \mid r_{b}=r}[\gamma]$ and $\mathcal{P}_{r_{b}}(r)$ denote the CDF of the SINR conditioned on $r_{b}=r$ and the PDF of the transmitter-receiver distance (given in Lemma 1), respectively.

\section{Numerical and Simulation Results}

In this section, we evaluate the performance of 3D deterministic random (Brownian) motion model. To confirm our framework, we use MC methods to obtain simulation result in different scenarios.

\subsection{Impact of number of antennas}

To gain insight into the effect of diffferent number of antennas, we provide results using deterministic model, stationary model and 3D Brownian model via MC simulation in Fig.1. A key point to note is that due to the deterministic model is the most ideal movement model and the 3D Brownian model is the most worst movement model, so in Fig.1 the deterministic model curves are the upper bound of reality and the 3D Brownian model curves are the lower bound of reality. Also, 
SE increases as the number of antennas increases and we can obtain the better performance of drone mobile networks by adjusting the number of antennas.

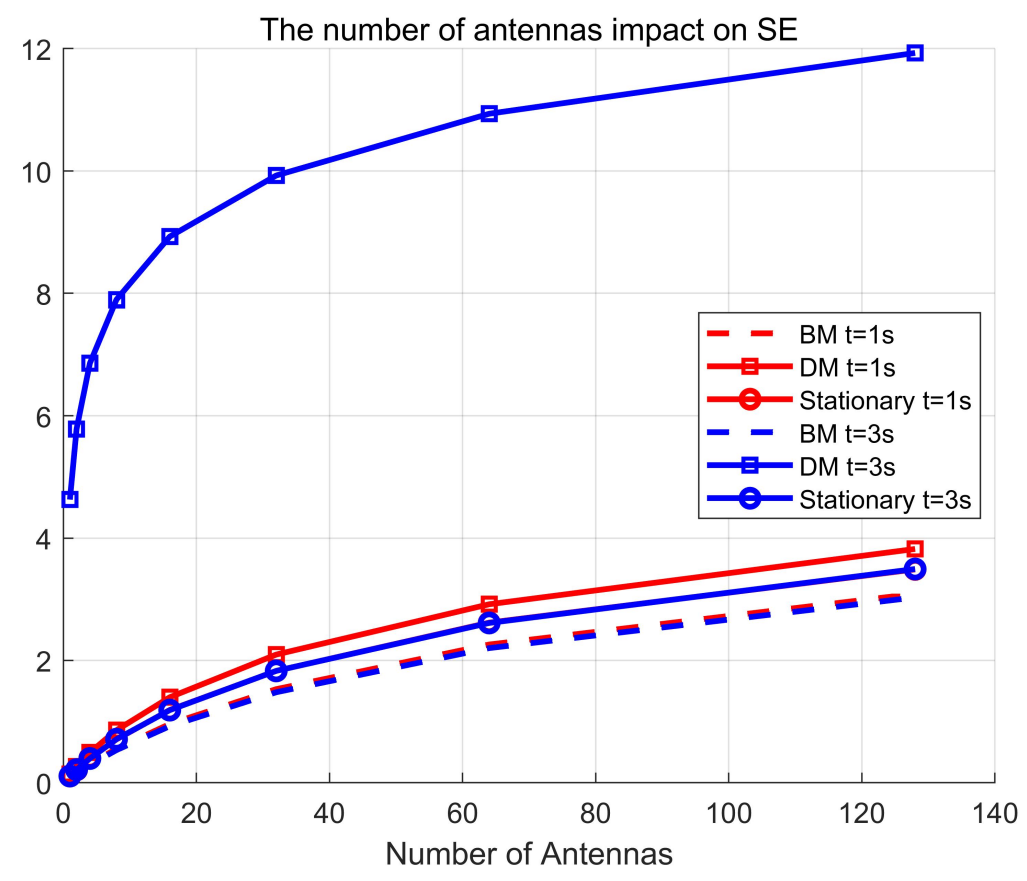

Fig. 1. Spectral efficiency against different number of antennas, $m=2, \mathrm{R}=100 \mathrm{~m}$, $\mathrm{D}=18 \mathrm{~m}, \lambda=10^{-5} / \mathrm{m}^{3}, v=3 \mathrm{~m} / \mathrm{s}, p=20 \mathrm{~W}$.

\subsection{Impact of Nakagami-m fading parameter}

We evaluate the Nakagami- $m$ fading parameter's impact on spectral efficiency. Fig.2 shows the drone mobile networks performance with different Nakagami$m$ fading parameter. We observe that when the Nakagami-m fading parameter increase s, some curve's SE decrease and the other curve's SE increase. The reason is that when the serving drone move three second baesd on deterministic movement model, the channel of serving drone $b$ becomes LOS, but other curve's channels are still NLOS (i.e., the channel function is different), and we can find 
Jiayi Huang et al.

that the performance of $\mathrm{SE}$ at time $t=3$ is better than time $t=0$. We can derive the SE of drone mobile networks in different channel cases.

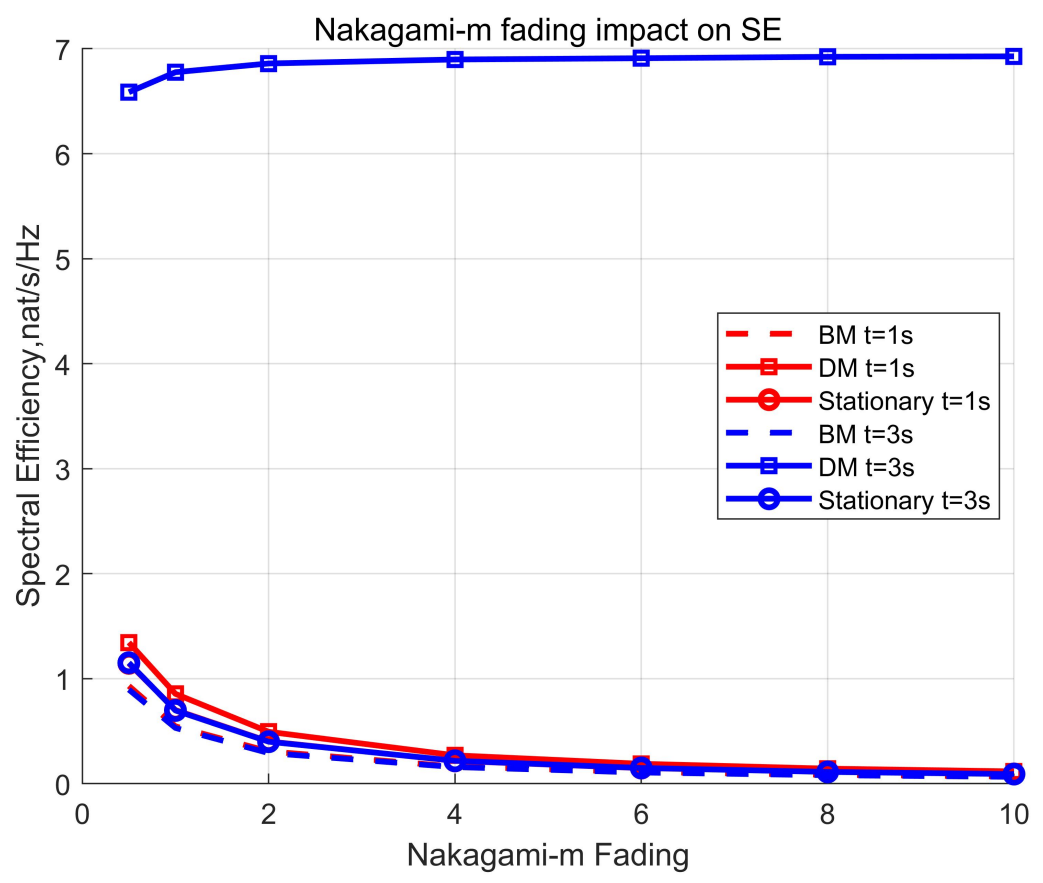

Fig. 2. The Nakagami-m fading impact on Spectral efficiency, $M=4, R=100 m, D=18 m$, $\lambda=10^{-5} / m^{3}, v=3 \mathrm{~m} / \mathrm{s}, p=20 \mathrm{~W}$.

\subsection{Impact of different drone velocity}

We explore the influence of different drone velocity under different movement strategies at time $t=0$ and time $t=3$ in Fig.3. A key point we can find that the drone velocity has a huge impact on $\mathrm{SE}$, especially on deterministic movement model and 3D Brownian movement model. So we can obtain the performance boundaries when we change the velocity of drone, but also need to consider the seurity issues at high velocity. 
System-Level Performance Analysis in 3D Drone Mobile Networks

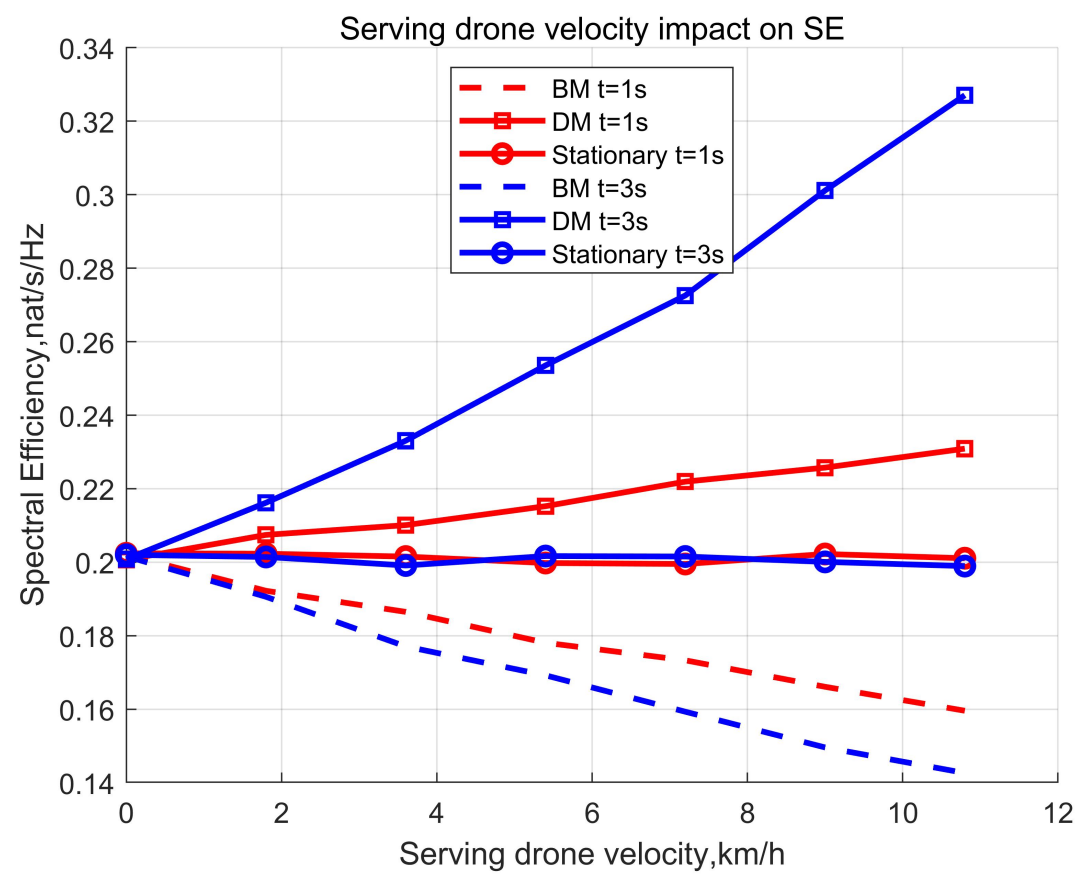

Fig. 3. The drone velocity impact on Spectral efficiency, $M=4, m=2, \mathrm{R}=100 \mathrm{~m}, \mathrm{D}=18 \mathrm{~m}$, $\lambda=10^{-5} / m^{3}, p=20 \mathrm{~W}$. 
Jiayi Huang et al.

\subsection{Impact of different deployment density}

We examine the influence of drone deployment density in improving drone mobile networks under various movement model and different time. Since the deployment density of drones largely determines the economic cost and network performance of drone mobile network, it is necessary to study the impact of deployment density on system performance. Fig.4 represents the performance of SE in different drone deployment density and we can find that there is a best deployment density around $\lambda=3 \times 10^{-5} / \mathrm{m}^{3}$. Therefore, in practical applications, we can optimize the system performance and cost by adjusting the deployment density.

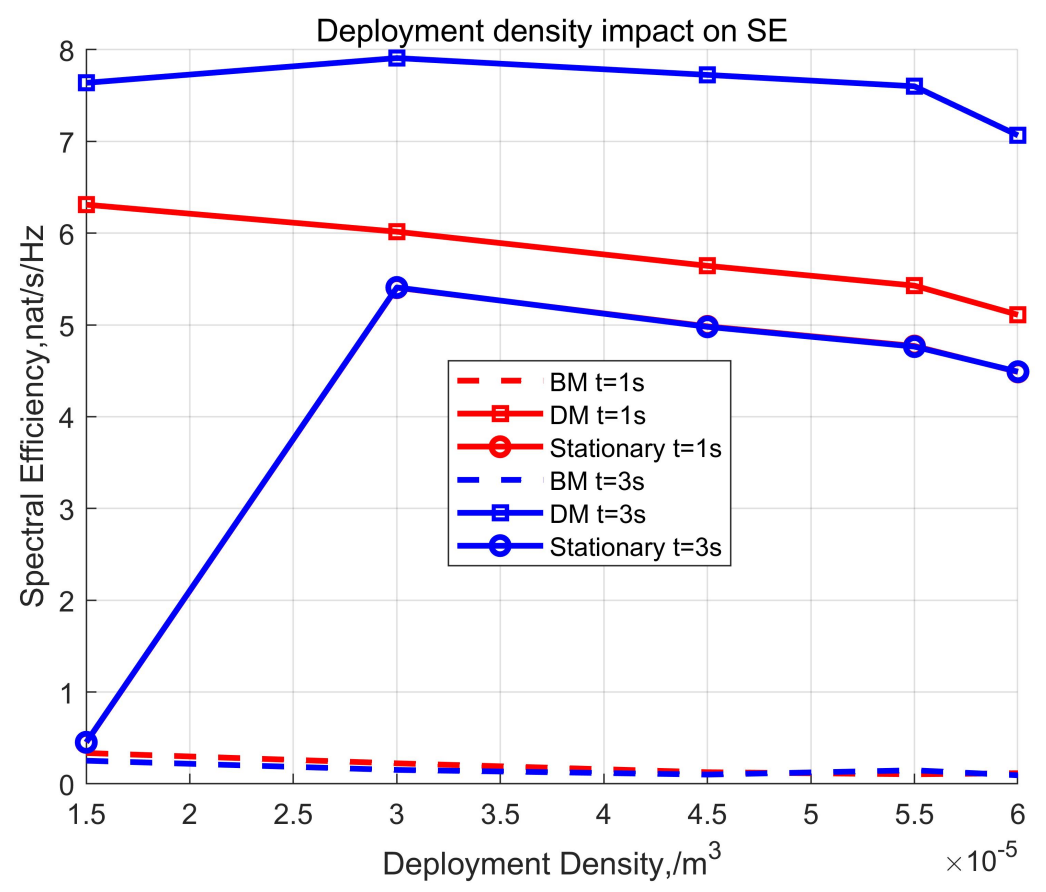

Fig. 4. The serving drone deployment density impact on Spectral efficiency, $M=4$, $m=2, \mathrm{R}=100 \mathrm{~m}, \mathrm{D}=18 \mathrm{~m}, v=3 \mathrm{~m} / \mathrm{s}, p=20 \mathrm{~W}$. 
System-Level Performance Analysis in 3D Drone Mobile Networks

\section{Conclusion}

This paper provides a system-level analysis of the drone mobile networks. In order to make the drone mobile networks model closer to the actual application scenario, this paper explores the feasibility and performance of the deterministic random (Brownian) motion model, and also explicitly account for some parameters of the drone networks, such as the impact of drone mobility based on deterministic random (Brownian) motion. Then we derive the expressions of SINR, average rate through the mathematical tools provided by stochastic geometry theory. We also validated the theoretical derivation by Monte Carlo simulation. The simulation results confirm the feasibility of the deterministic random (Brownian) motion model. In the follow-up study, we will use this as a basis to analyze the performance of drone mobile networks after the introduction of MIMO.

\section{References}

1. Esma Turgut and M Cenk Gursoy. Downlink analysis in unmanned aerial vehicle (uav) assisted cellular networks with clustered users. IEEE Access, 6:36313-36324, 2018.

2. Vishnu Vardhan Chetlur and Harpreet S Dhillon. Downlink coverage analysis for a finite 3-d wireless network of unmanned aerial vehicles. IEEE Transactions on Communications, 65(10):4543-4558, 2017.

3. Wenqiang Yi, Yuanwei Liu, Arumugam Nallanathan, and George K Karagiannidis. A unified spatial framework for clustered uav networks based on stochastic geometry. In 2018 IEEE Global Communications Conference (GLOBECOM), pages 1-6. IEEE, 2018.

4. Mohamed Alzenad and Halim Yanikomeroglu. Coverage and rate analysis for unmanned aerial vehicle base stations with los/nlos propagation. In 2018 IEEE Globecom Workshops (GC Wkshps), pages 1-7. IEEE, 2018. 
Jiayi Huang et al.

5. David López-Pérez, Ming Ding, Huazhou Li, Lorenzo Galati Giordano, Giovanni Geraci, Adrian Garcia-Rodriguez, Zihuai Lin, and Mahbub Hassan. On the downlink performance of uav communications in dense cellular networks. In 2018 IEEE Global Communications Conference (GLOBECOM), pages 1-7. IEEE, 2018.

6. Shangwei Zhang and Jiajia Liu. Analysis and optimization of multiple unmanned aerial vehicle-assisted communications in post-disaster areas. IEEE Transactions on Vehicular Technology, 67(12):12049-12060, 2018.

7. Sung Nok Chiu, Dietrich Stoyan, Wilfrid S Kendall, and Joseph Mecke. Stochastic geometry and its applications. John Wiley \& Sons, 2013.

8. Arman Shojaeifard, Kai-Kit Wong, Marco Di Renzo, Gan Zheng, Khairi Ashour Hamdi, and Jie Tang. Massive mimo-enabled full-duplex cellular networks. IEEE Transactions on Communications, 65(11):4734-4750, 2017.

9. Akram Al-Hourani, Sithamparanathan Kandeepan, and Abbas Jamalipour. Modeling air-to-ground path loss for low altitude platforms in urban environments. In 2014 IEEE global communications conference, pages 2898-2904. IEEE, 2014.

10. Sunil Srinivasa and Martin Haenggi. Distance distributions in finite uniformly random networks: Theory and applications. IEEE Transactions on Vehicular Technology, 59(2):940-949, 2010.

11. Ponnambalam Kumaraswamy. A generalized probability density function for double-bounded random processes. Journal of Hydrology, 46(1-2):79-88, 1980.

12. Jihong Park, Sang Yeob Jung, Seong-Lyun Kim, Mehdi Bennis, and Mérouane Debbah. User-centric mobility management in ultra-dense cellular networks under spatio-temporal dynamics. In 2016 IEEE Global Communications Conference (GLOBECOM), pages 1-6. IEEE, 2016.

13. ATHANASIOS. PAPOULIS. Probability, random variables, and stochastic processes. 1985. 